\title{
Lasing from dye-doped photonic crystals with graded layers in dichromate gelatin emulsions
}

\author{
Mang Hin Kok, Weixin Lu, Jeffrey Chi Wai Lee, Wing Yim Tam, ${ }^{a)}$ George K. L. Wong, \\ and C. T. Chan \\ Department of Physics and William Mong Institute of Nano Science and Technology, The Hong Kong \\ University of Science and Technology, Clear Water Bay, Kowloon, Hong Kong, People's Republic of China
}

(Received 3 December 2007; accepted 18 March 2008; published online 17 April 2008)

\begin{abstract}
We report on optically pumped lasing from dye-doped, graded-spacing layer structures of dichromate gelatin emulsions fabricated using two-beam holographic interference. The graded layers exhibited deep and wide photonic band gaps. Multimode lasing with both a low threshold and a high quality factor was observed at the band edge of the photonic band gap. We modeled the emissions from the dye-doped graded layer system using a finite difference time domain technique and achieved good agreement with experimental results. (C) 2008 American Institute of Physics. [DOI: 10.1063/1.2907488]
\end{abstract}

Photonic crystals exhibiting band gaps in which electromagnetic wave propagation is not possible have attracted much interest due to the potential that they can manipulate photons in optical microdevices. ${ }^{1}$ Among the many unique properties of photonic crystals, the control of the photonic density of states has a direct effect on the spontaneous emission of photons, which is an important capability in optoelectronics devices such as lasers, ${ }^{2}$ light emitting diodes, ${ }^{3}$ microwaveguides, ${ }^{4}$ and many other promising photonic devices. ${ }^{5}$ It has been proposed that the enhancement of the spontaneous emissions in one-dimensional (1D) photonic crystals doped with gain materials is possible because of the localization at the band gaps and the high density of states at the band edges. ${ }^{6}$ The realization of this enhancement was first demonstrated in GaAs light-emitting diodes sandwiched between stacks of 1D Bragg reflectors. ${ }^{7}$ Since then, lasing from dye-doped polymeric multilayers using distributed feedback and defect modes has been observed. ${ }^{8,9}$ Recently, studies on band-edge and defect-mode lasing in dye-doped liquid crystals have attracted interest. ${ }^{10}$ In these previous studies, equally spaced layers were employed in the systems. Lasing from 1D layers with gradually changing spacings (graded layers) is expected to be possible as well.

One-dimensional stacks have been fabricated using a time-consuming layer-by-layer spin-coating method. ${ }^{7-9}$ Holographic lithography that employs the interference of multiple coherent beams is more efficient and has been used to fabricate two-dimensional as well as three-dimensional microstructures in photoresists. ${ }^{11}$ Furthermore, high-resolution holographic gelatin emulsions can be used to record the interference patterns. ${ }^{12,13}$ One advantage to using holographic gelatin emulsions is that the structure resulting from the interference is self-supporting inside the gelatin. It was further demonstrated that, with a volume hologram made from dichromate gelatin (DCG), highly efficient and wide-bandgap 1D layer structures could be achieved. ${ }^{14}$ The wide-bandgap results from the graded spacing of the 1D layers obtained by the differential swelling of the gelatin during the development process. ${ }^{14}$ Here, we show that lasing from dyedoped 1D graded layers in DCG holographic emulsions fab-

\footnotetext{
a) Author to whom correspondence should be addressed. Electronic mail: phtam@ust.hk. Tel: 852-2358-7490. FAX: 852-2358-1652.
}

ricated using two-beam interference is possible. Multimode lasing with both a low threshold and a high quality factor was observed at the band edge of the photonic band gap. We modeled the emissions from the graded layer system using a finite difference time domain (FDTD) technique and achieved good agreement with experimental results.

The experimental setup was similar to that reported earlier. ${ }^{14}$ A beam from an argon ion laser, with a $488 \mathrm{~nm}$ wavelength $(\lambda), 2-3 \mathrm{~mW}$ of power, and $9 \mathrm{~mm}$ in diameter, normally entered the DCG emulsion from the substrate side of the holographic plate and interfered with its reflection via a mirror in contact with the emulsion through a thin layer of matching fluid to form a layered structure of spacing $d$ $=\lambda / 2 n$ inside the gelatin (where $n$ is the refractive index of the DCG). After $30 \mathrm{~s}$ exposure, the plate was gently rinsed in a $15{ }^{\circ} \mathrm{C}$ water bath for $15 \mathrm{~s}$ to remove the matching fluid before being soaked in another water bath at the same temperature containing a $0.001 \mathrm{gm} / \mathrm{ml}$ organic dye made from Rhodamine 590 for 30 min enabling the dye molecules to diffuse deep into the layers of the gelatin. After the swelling step, the DCG plate was hardened by baking in an oven at $100{ }^{\circ} \mathrm{C}$ for $60 \mathrm{~min}$. After it cooled to room temperature, the plate was rinsed under running tap water for $3 \mathrm{~min}$ to remove any residual dichromate. The plate was then dehydrated by soaking it in $50 \%, 75 \%$, and $100 \%$ isopropanol baths containing the same Rhodamine 590 dye concentration as in the swelling water bath at 20 to $40{ }^{\circ} \mathrm{C}$ for $3 \mathrm{~min}$ each. After dehydration, the plate was baked again at $100{ }^{\circ} \mathrm{C}$ for $60 \mathrm{~min}$. Finally, to protect the structure against moisture, a $0.2 \mathrm{~mm}$ thick cover glass was placed on the top of the DCG emulsion and sealed with wax. The DCG emulsion showed swelling depending on the dehydration temperature. ${ }^{14}$ Rhodamine 590 was used because its fluorescence is the highest at $\sim 560 \mathrm{~nm}$, which is near the shortwavelength (left) band edge of the stop bands in the layer structures fabricated under the above conditions.

The dye-doped DCG samples were characterized by measuring the reflectance and transmittance of white light normally shone from either side of the DCG emulsion as shown in the top-left inset of Fig. 1(a). An optical fiber was used to couple the reflected or transmitted light to a spectrometer. Figures 1(a) and 1(b) show the reflectance and transmittance for two samples dehydrated at temperatures 37 


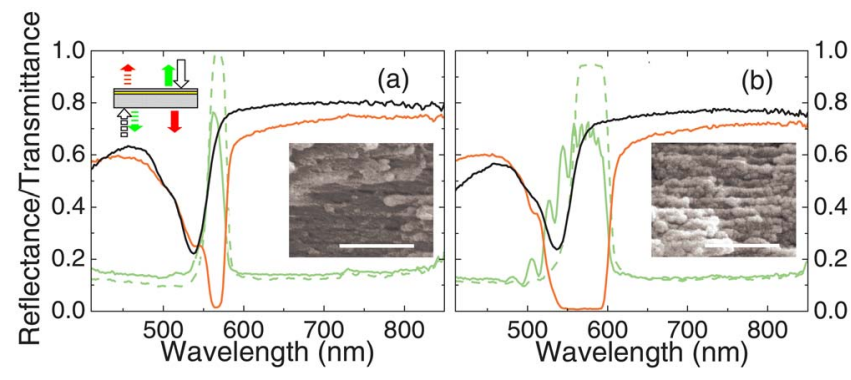

FIG. 1. (Color online) Normal reflectance (green) and transmittance (red) of dye-doped, layer-structured DCG samples. The solid and dotted lines are obtained by white-light incidence on the gelatin and substrate sides, respectively, as illustrated in the upper-left schematic inset in (a). The black lines show the transmittance in the unexposed regions. The lower-right insets show close-up, cross-sectional SEM images of the layered structures in the upper-middle regions of the samples. The white scale bars are $1 \mu \mathrm{m}$.

and $40{ }^{\circ} \mathrm{C}$, respectively. The samples exhibited high reflection efficiency from either side of the DCG emulsions and, more importantly, very low (less than $1 \%$ ) transmittance at the stop bands with a full width at half maximum (FWHM) of $\sim 25 \mathrm{~nm}$ and $\sim 70 \mathrm{~nm}$ for (a) and (b), respectively. The deep and wide stop band gaps were results of the graded layer structures. This was confirmed by the scanning electron microscope (SEM) images as shown in the insets of Figs. 1(a) and 1(b) with spacings of about $183-190 \mathrm{~nm}$ and $180-200 \mathrm{~nm}$ from the top air-gelatin side to the bottom gelatin-substrate side, respectively. ${ }^{14}$ Also shown in Fig. 1 are the transmittances of the dye-doped gelatin from the unexposed regions of the two samples. Absorption of the dye at $\sim 540 \mathrm{~nm}$ is clearly shown.

We used a $\mathrm{Nd}^{3+}$-doped yttrium aluminium garnet laser at $532 \mathrm{~nm}$, with $35 \mathrm{ps}$ wide pulses and a $10 \mathrm{~Hz}$ repetition rate, as the pumping source to study the photoluminescence (PL) of the dye-doped DCG samples. After passing through a set of polarizers and a half-wave plate, the laser was focused on a $0.8 \mathrm{~mm}$ spot of the sample at an incident angle of $\phi$ $\left(\sim 62^{\circ}\right)$ so that the pumping beam could pass through the sample to excite the embedded dye molecules inside the gelatin, as shown in the inset of Fig. 2. The PL in the forward direction was collected by a lens focused onto an optical fiber that was coupled to a linear charge-coupled device spectrometer. The sample was excited either with the gelatin side or the substrate side facing the pumping beam, equivalent to measuring the PL from the substrate side or the gelatin side, respectively. Figures 2(a) and 2(c) show the PL at low pumping energies, normalized to that of the unexposed regions at long wavelengths, for the samples described in Figs. 1(a) and 1(b), respectively, from both the substrate and gelatin sides. Also shown in Fig. 2 are the stop bands of the dye-doped DCG layered structures and the PL in the unexposed regions (blue lines) of the two samples. We note that the PL was suppressed inside the stop bands of the layered structures but it was enhanced at the band edges. Lasing was observed in both samples at higher pumping energies as shown in Figs. 2(b) and 2(d). We observed lasing from both the substrate (pink lines) and gelatin sides (orange lines) of the sample described in Fig. 1(a) but only from the substrate side of the sample described in Fig. 1(b). This was because the left band edges of the stop bands in the sample described in Fig. 1(a) on both the substrate (dotted green lines) and gelatin (solid green lines) sides were close to the peak PL of the dye (blue line) at $\sim 555 \mathrm{~nm}$, where the spontaneous emis-

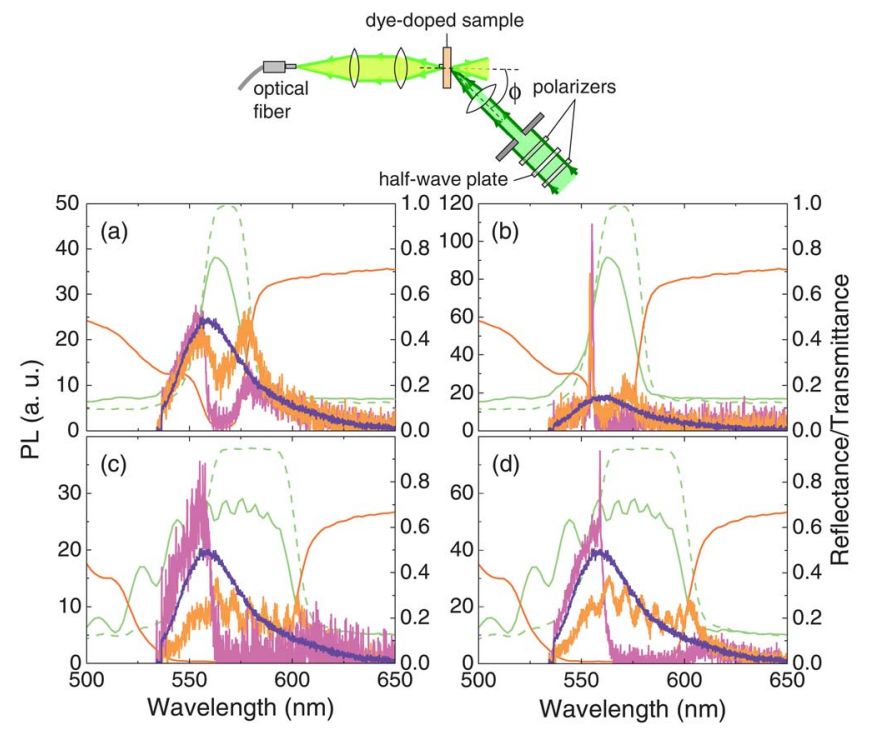

FIG. 2. (Color online) PL spectra of the substrate-side (pink) and gelatinside (orange) emissions of dye-doped DCG samples as depicted in Fig. 1, obtained by using the schematic setup in the top inset. Also shown are the optical spectra (green lines for reflection and red lines for transmission) at the stop bands. The pumping energies (in $\mu \mathrm{J} /$ pulse) are (a) 0.63 (pink), 0.50 (orange); (b) 0.99 (pink), 1.00 (orange); (c) 0.41 (pink), 0.66 (orange); (d) 1.25 (pink), 1.23 (orange). The blue lines are the PL spectra of Rhodamine 590-doped DCG in the unexposed regions.

sion was maximized at the band edges in both cases. However, this was not the case for the sample described in Fig. 1(b), where only the left band edge of the substrate-side stop band was close to the peak PL, leading to one-sided lasing even when it was pumped with very high energy.

There was a critical pumping energy value for lasing to occur. Figure 3 shows the PL spectra of different pumping energies for the sample described in Fig. 1(b). The pumping

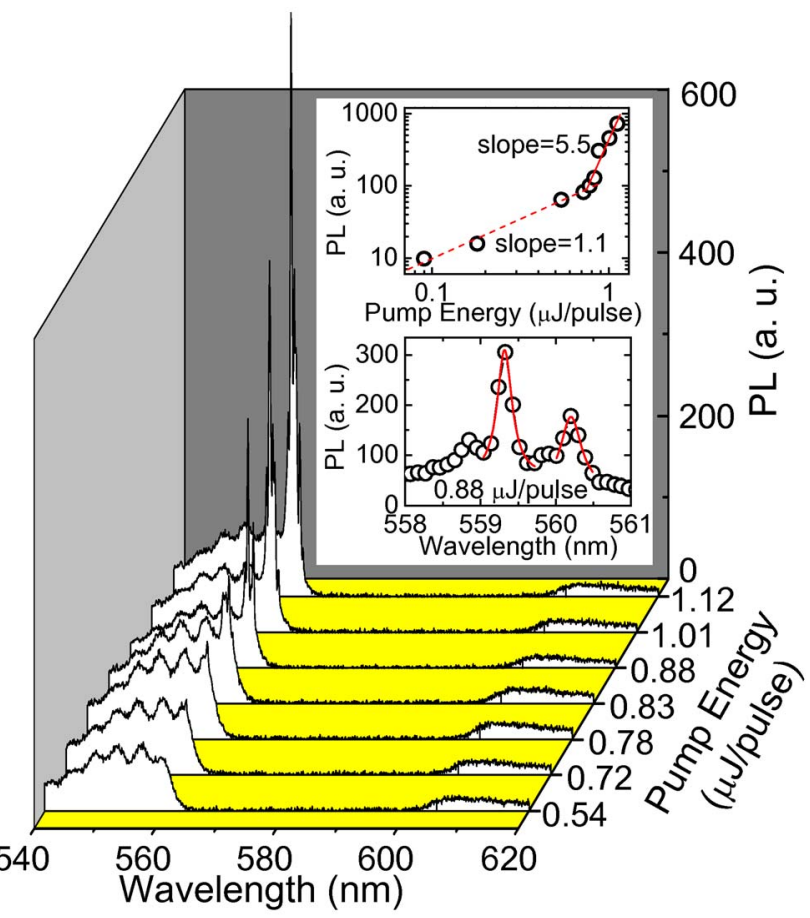

FIG. 3. (Color online) The PL spectrum of the sample in Fig. 1(b) as a function of the pumping energy. The upper inset shows the measured peak emission intensity (circles) at $559.3 \mathrm{~nm}$. The dotted and solid lines are linear fits for pumping energies below and above the lasing threshold, respectively. The lower inset shows the multimode lasing from Fig. 1(b).

to AIP license or copyright; see http://apl.aip.org/apl/copyright.jsp 

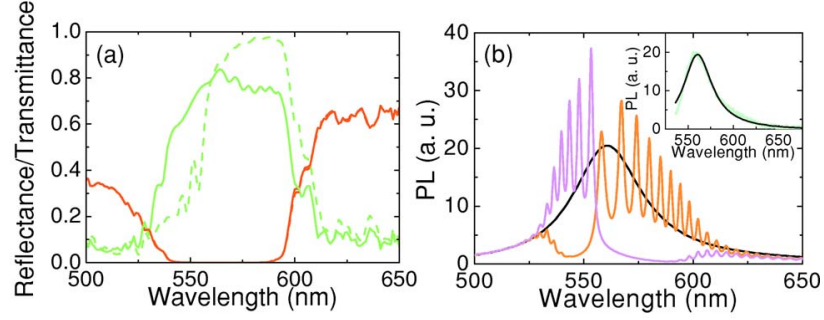

FIG. 4. (Color online) (a) Normal reflectance (green) and transmittance (red) obtained from the transfer matrix model. The solid and dotted lines are obtained by light incidence on the gelatin and substrate sides, respectively. (b) Simulated emission spectra for the dye-doped DCG samples from the substrate (pink) and gelatin (orange) sides using the layer configuration in (a) in comparison with the simulated emission spectrum of dye in free space (black).

energy at the onset of lasing was $\sim 0.83 \mu \mathrm{J} /$ pulse, which was low compared with the energies reported for other 1D lasing systems. ${ }^{15,16}$ The top inset of Fig. 3 shows the peak PL intensity in relation to the pumping energy. Before lasing, the PL intensity had an almost linear relationship with the pumping energy (dashed line) as indicated by the slope of $\sim 1.1$, but this relationship switched to having a power-law dependence (solid line) with a slope of $\sim 5.5$ after lasing. Furthermore, multiple lasing lines with $\sim 1 \mathrm{~nm}$ spaces were observed as shown in the expanded scale in the lower inset of Fig. 3 for pumping energies slightly beyond the critical value. We believe that these lines indicate lasings from different layered sections inside the gelatin. Furthermore, the FWHM was found, by fitting the lasing peaks shown in the lower inset of Fig. 3 (solid red lines), to be less than $0.3 \mathrm{~nm}$ with a quality factor, defined as $\lambda_{\text {peak }} / \mathrm{FWHM}$, of about $\sim 2000$, which was comparable to other 1D lasing systems. ${ }^{16}$

We used an effective medium approach to model the dye-doped, graded-layer structure (DGLS) sample and a transfer matrix to calculate the reflectance and transmittance. ${ }^{14}$ We used a previously established calculation method $^{14}$ on a layered structure with graded spacing of 181-202 nm (top to bottom). Each layer was approximated as a two-component composite, one was a dense gelatin part $a$ and the other was a less-dense gelatin part $b$. The dielectric constant for the dense part $a$ was kept constant with $\varepsilon_{a}$ $=2.262$ and no absorption. The real part of the dielectric constant for the less-dense part $b, \operatorname{Re}\left(\varepsilon_{b}\right)$, varied from 1.982 to 1.621 (top to bottom) and the imaginary part, $\operatorname{Im}\left(\varepsilon_{b}\right)$, was fixed at 0.008 to incorporate the gelatin absorption. To account for the absorption from the Rhodamine dye, 50\% $(60 \%)$ of the measured dye absorption was included in the reflectance at the substrate (gelatin) side while $100 \%$ was included in the transmittance. The results were shown in Fig. 4(a) for the sample described in Fig. 1(b). The agreement of the band edges and band gaps with the experimental data is excellent. We also calculated the emission spectrum from the DGLS using the FDTD scheme. ${ }^{17}$ For simplicity, we placed identical planar light emitting sources at the center of each layer to simulate a uniform distribution of the light-emitting dye inside the DGLS. The dye distributions with gradients gave qualitatively similar results. In addition, we employed a Lorentzian frequency profile, obtained by fitting the dye photoluminescence taken from Fig. 2(c) (cyan line) as shown in the inset of Fig. 4(b) (black line) for the planar source. The result of this FDTD simulation could not predict the onset of lasing, as we assumed at the outset that the layers were lightemitting sources, but the simulation did give information on the effect of the scattering due to the layered structures. We further assumed that the light emitted from different layers of the DGLS was incoherent so that the intensity of the light from all layers added up rather than coming from the field itself. Hence, the simulated PL emission was a summation of the intensities obtained from many calculations for different light-emitting layers. The FDTD results are shown in Fig. 4(b). We see that the emissions, suppressed inside the stop bands, from the two sides of the DGLS are indeed different, and the results capture all the salient features of the experimental observations. This was expected as the layer spacings on the two ends of the DGLS were of different thicknesses. On the gelatin side, the layer spacings were on average narrower, and the band gap shifted to a higher frequency, thus, allowing emission on the lower frequency side. The emission from the substrate side was stronger as the layer spacings were on average wider there and thus had a lower frequency band gap. The calculated results agreed well with the experimental results.

To conclude, we fabricated dye-doped graded planar structures in a DCG holographic gelatin emulsion using a two-beam interference technique. High efficiencies and wide stop bands were obtained such that lasing with a lowthreshold pumping energy and narrow FWHM was observed when the system was optically excited. FDTD calculations were in good agreement with the experimental results.

Support from Hong Kong RGC Grants Nos. HKUST603405, HKUST602606, and HKUST3/06C is gratefully acknowledged. The computation resources were supported by the Shun Hing Education and Charity Fund.

\footnotetext{
${ }^{1}$ See, e.g., Photonic Band Gap Materials, edited by C. M. Soukoulis (Kluwer, Dordrecht, 1996).

${ }^{2}$ See, e.g., A. E. Siegman, Lasers (University Science, Palo Alto, 1986).

${ }^{3}$ M. D. Tocci, M. J. Bloemer, M. Scalora, J. P. Dowling, and C. M. Bowden, Appl. Phys. Lett. 66, 2324 (1995).

${ }^{4}$ B. Wang, J. Jiang, and G. P. Nordin, Opt. Express 12, 3313 (2004).

${ }^{5}$ Y. Dumeige, P. Vidakovic, S. Sauvage, I. Sgnes, and J. A. Levenson, Appl. Phys. Lett. 78, 3021 (2001).

${ }^{6}$ J. P. Dowling, M. Scalora, M. J. Bloemer, and C. M. Bowden, J. Appl. Phys. 75, 1896 (1994).

${ }^{7}$ M. D. Tocci and M. Scalora, Phys. Rev. A 53, 2799 (1996).

${ }^{8}$ T. Komikado, S. Yoshida, and S. Umegaki, Appl. Phys. Lett. 89, 061123 (2006).

${ }^{9}$ J. Yoon, W. Lee, J. Caruge, M. Bawendi, E. L. Thomas, S. Kooi, and P. N. Prasad, Appl. Phys. Lett. 88, 091102 (2006).

${ }^{10}$ Y. Matsuhisa, Y. Huang, Y. Zhou, S. T. Wu, R. Ozaki, Y. Takao, A. Fujii, and M. Ozaki, Appl. Phys. Lett. 90, 091114 (2007).

${ }^{11}$ M. Campbell, D. N. Sharp, M. T. Harrison, R. G. Denning, and A. J. Turberfield, Nature (London) 404, 53 (2000).

${ }^{12}$ J. M. Kim, B. S. Choi, S. I. Kim, J. M. Kim, H. I. Gjelkhagen, and N. J. Phillips, Appl. Opt. 40, 622 (2001).

${ }^{13}$ M. H. Kok, R. Ma, J. C. W. Lee, W. Y. Tam, C. T. Chan, and P. Sheng, Phys. Rev. E 72, 047601 (2005).

${ }^{14}$ R. Ma, J. Xu, and W. Y. Tam, Appl. Phys. Lett. 89, 081116 (2006).

${ }^{15}$ S. M. Morris, A. D. Ford, M. N. Pivnenko, and H. J. Coles, J. Appl. Phys. 97, 023103 (2005).

${ }^{16}$ Y. Zhou, Y. Huang, A. Rapaport, M. Bass, and S. Wu, Mol. Cryst. Liq. Cryst. 454, 377 (2006).

${ }^{17}$ A. Farjadpour, D. Roundy, A. Rodriguez, M. Ibanescu, P. Bermel, J. D. Joannopoulos, S. G. Johnson, and G. W. Burr, Opt. Lett. 31, 2972 (2006).
} 